\title{
Single-user performance of direct-sequence code-division multiple-access using relay diversity and power allocation
}

\author{
W. Fang L.-L. Yang L. Hanzo
}

School of Electronics and Computer Science, University of Southampton, Southampton SO17 1BJ, UK

E-mail: Ily@ecs.soton.ac.uk

\begin{abstract}
In this contribution, the authors investigate the single-user-bound performance of a direct-sequence code-division multiple-access (DS-CDMA) system, where one source mobile terminal (MT) communicates with its base-station (BS) with the assistance of multiple relays. The authors assume that the communications channels experience both propagation pathloss and fast fading, and that the channels from the source MT to the BS and relays as well as that from the relays to the BS may experience different fast fading modelled correspondingly by Nakagami- $m$ distributions. In the study, they assume both the single-user combining using maximal ratio combining and the multiuser combining, which are derived based on the maximum signal-tointerference-plus-noise ratio criterion. The bit-error-rate (BER) performance of the DS-CDMA is investigated associated with considering the locations of the relays as well as the power-allocation among the source MT and relays. From the study and simulation results, it can be shown that the achievable BER performance of the DS-CDMA depends on the locations of the relays and also on the power-allocation among the source MT and relays. When the relays of a source MT are chosen from a different area, the power-allocation should also be adjusted correspondingly in order to achieve the minimum BER. Furthermore, when optimum powerallocation is assumed, the BER performance of the DS-CDMA can be significantly improved, when increasing the number of relays assisting the source MT.
\end{abstract}

\section{Introduction}

It is well-known that signals transmitted over wireless communication channels suffer from fading, which may be efficiently mitigated by exploiting various types of diversity in time-, frequency- and/or spacedomain. In wireless communications, spatial diversity is particularly attractive, since it is capable of offering spectral efficiency without incurring an expenditure of transmission time and bandwidth [1]. Spatial diversity can usually be achieved with the assistance of deploying multiple antennas at transmitter and/or receiver [2-4]. In cellular wireless communication systems, multiple antennas are desirable for deployment at base stations (BSs), in order to achieve the downlink transmit diversity. However, the transmit diversity that depends on multiple transmit antennas is not directly applicable to the uplink transmission, due to the mobile unit's size limitation. Recently, cooperative diversity in wireless communication systems with distributed nodes or users has attracted wide attention and research, as can be seen, for example, in [5-8]. This is because the capacity of a wireless communications system may be significantly improved through cooperation among the distributed nodes or users. Specifically, in a wireless system with distributed mobile terminals (MTs), a set of MTs may share their transmit antennas in order to create a virtual transmit antenna set for achieving the transmit diversity $[5,6,9,10]$. This type of transmit diversity is usually referred to as relay diversity.

The performance of the wireless systems using relay diversity has been widely investigated in the literature, 
for example, in $[9-11,12]$, when assuming that there exists no interference among the relays, even when the relays access the wireless channel at the same time. However, in practical wireless communication systems such as in cellular direct-sequence codedivision multiple-access (DS-CDMA) systems, there usually exists multiple-access interference (MAI) among the MTs, when they access the wireless channels using the same frequency band at the same time. In a relay-assisted DS-CDMA system, MAI may exist at the relays as well as at the BS. The MAI can be suppressed with the aid of multiuser detection (MUD) as suggested in [13, 14]. However, in [13, 14] it is assumed that each MT has only one relay. Furthermore, the impact of relays' locations on the achievable bit-error-rate (BER) performance has not been investigated in [13]. The power allocation issue has not been considered in [14]. In this contribution, we investigate the single-user BER performance of the uplink DS-CDMA, when one MT is assisted by several other MTs acting as relays, in order to achieve the relay diversity. Specifically, we assume a lowcomplexity amplify-and-forward (AF) cooperation scheme, where signals at the relays are first despread using the source transmitter's spreading code and then forwarded to the BS using the relays' spreading codes. In our investigation, both the propagation pathloss and fast fading [15] are considered, in order to demonstrate the fact that appropriately selected relays can be beneficial to saving the transmission power for achieving a higher power-efficiency. For fast fading, we assume a generalised Nakagami fading channel model [16], where signals from the source transmitter to the relays and that from the relays to the BS may experience different fading. At the BS the signals received from the source $\mathrm{MT}$ and relays are combined according to either a single-user combining (SUC) or a multiuser combining (MUC) scheme. The SUC scheme maximises the output SNR without taking into account the interference among the relays, while the MUC scheme maximises the signal-tointerference-plus-noise ratio (MSINR) and suppresses the interference among the relays.

In this contribution, the single-user BER performance of the DS-CDMA system is investigated, when the power allocation among the source transmitter and relays is applied, in order to demonstrate the advantages of using cooperation diversity. By contrast, most previous study, for example, in [9-11], the power allocation has not been considered. Our analysis and simulation results show that the relays of a given MT should be chosen from certain geographical area, in order to achieve the best available BER performance. Furthermore, it can be shown that the BER performance of the DS-CDMA system can be significantly improved, when optimum power allocation among the transmitter and relays is utilised. In other words, our investigation shows that the cooperation among the MTs in a wireless network is highly efficient for reducing the total radiated power necessitated to ensure the delivery of information with desired quality of service (QoS).

Note that, the reason for us to consider only the case of single-user multi-relays is that from the single-user performance results we can gain insight into the achievable performance of the DS-CDMA using relays, when the DS-CDMA system supports multiple users associated with employing advanced MUD. Furthermore, our approaches in this contribution can be extended to the DS-CDMA supporting multiple users, which constitutes our future work.

The rest of the paper is organised as follows. Section 2 describes the system model, including the transmitted signal, channel model, cooperation strategy and receiver model. In Section 3 the signal detection is considered. Section 4 shows the simulation results, and finally, in Section 5, we summarise our conclusions.

\section{System description}

In this section, we present the DS-CDMA system using relay diversity. The transmitted signals at the source MT and relays, the cooperation strategy at the relays, the receiver model as well as the channel model are considered, respectively.

\subsection{Transmitted signal at source MT}

In our considered DS-CDMA, there is one source MT, say MT $k$, communicating with the BS with the assistance of $L$ relays, which are also MTs. The signal transmitted by the $k$ th MT is a typical DS-CDMA signal and its complex baseband equivalent representation can be expressed as

$$
s_{k}(t)=\sqrt{2 P_{k t}} x_{k}(t) c_{k}(t)
$$

where $P_{k t}$ represents the transmitted power of MT $k$ on the real or imaginary axis, $x_{k}(t)$ represents the transmitted data waveform, which can be expressed as $x_{k}(t)=\sum_{n=0}^{\infty} x_{k}[n] P_{T}(t-n T)$, where we assume $E\left[\left|x_{k}\right|^{2}\right]=1, T$ represents the time duration of the source MT transmitting a symbol, $P_{T}(t)$ represents the rectangular waveform, which is defined as $P_{T}(t)=1$ if $0 \leq t<T$ and $P_{T}(t)=0$, otherwise. Furthermore, in $(1), \quad c_{k}(t)$ represents the binary DS spreading waveform, which can be expressed as $c_{k}(t)=\sum_{n=0}^{\infty} c_{k n} \psi_{T_{c}}\left(t-n T_{\mathrm{c}}\right)$, where $T_{\mathrm{c}}$ represents the chip-duration, $c_{k n} \Leftarrow\{-1,+1\}, \quad \psi_{T_{c}}(t)$ is the chipwaveform, which is defined within $\left[0, T_{\mathrm{c}}\right)$ and normalised to satisfy $\int_{0}^{T_{\mathrm{c}}} \psi_{T_{\mathrm{c}}}(t) \mathrm{d} t=T_{\mathrm{c}}$. Additionally, we define $N=T / T_{\mathrm{c}}$, which is the spreading factor. 


\subsection{Cooperation strategy and signal processing at relays}

The kth MT communicates with the BS assisted by a cluster of $L$ relays, as shown in Fig. 1. We assume that the distance between any two relays within a cluster does not exceeds 20 wavelengths. In this case, the propagation pathlosses from the source transmitter to the $L$ relays and that from the $L$ relays to the BS can be treated as the same [15]. We also assume that any two relays within a cluster are sufficiently separated ( $>$ several wavelengths), so that the channels in the context of any two relays are uncorrelated for achieving the maximum diversity. Furthermore, we assume that the relays do not have their own data to transmit. As shown in Fig. 1, for the sake of convenience, we define the direct channel (D-channel) as the channel connecting MT $k$ with the BS. The relay channel (R-channel) represents a channel from MT $k$ through a relay to the BS. Furthermore, the $\mathrm{R}$-channel includes the channel connecting the $k$ th MT to the relay and that connecting the relay to the BS, where the former is referred to as the TR-channel, while the latter as the RB-channel.

Throughout the paper, we assume that the source MT and the MTs acting as relays cannot transmit and receive signals simultaneously, and the cooperation scheme is based on time-division (TD). Specifically, a symbol duration is divided into two time-slots, the source MT transmits using the first time-slot, whereas the $L$ relays use the second time-slot to transmit the signals received from the $k$ th $\mathrm{MT}$ in the first time-slot to the BS. For the sake of focusing our attention on relay diversity alone, we consider here frequency nonselective fading channels. However, the results can be directly applied to frequency-selective fading channels, when orthogonal frequency-division multiplexing (OFDM) is invoked to convert the frequency-selective fading channel to a number of subchannels that are free from frequency-selective fading. Furthermore, we assume that the transmitted signals can be perfectly synchronised whenever necessary.

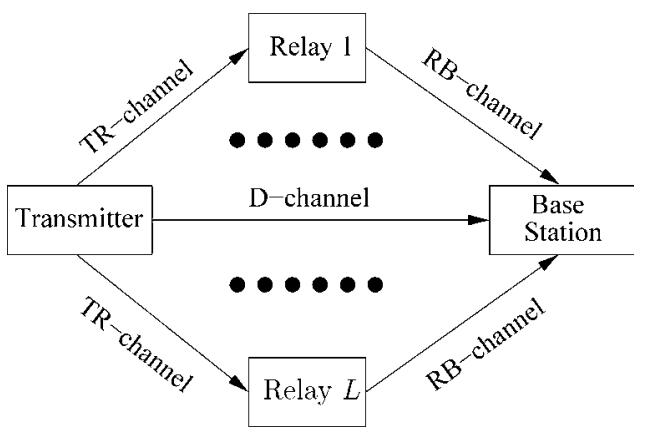

Figure 1 Channels in a relay-assisted DS-CDMA, where one transmitter is assisted by $L$ relays
Let the complex baseband equivalent signal received by the lth relay within the first timeslot of the $n$th symbol duration be expressed as

$$
\begin{array}{r}
r_{l}^{(k)}(t)=\sqrt{2 P_{l}^{(k)}} h_{l}^{(k)}{ }_{x_{k}}[n] c_{k}(t)+n_{l}^{(k)}(t), \\
l=1,2, \ldots, L
\end{array}
$$

where $P_{l}^{(k)}$ represents the received power of relay $l$ from the $k$ th user after taking into account the pathloss of the $l$ th TR-channel, $h_{l}^{(k)}$ represents the fading gain of the lth TR channel, while $n_{l}^{(k)}(t)$ represents the complex baseband equivalent Gaussian noise, which has mean zero and a variance of $N_{0}$ per dimension.

At the $l$ th, $l=1,2, \ldots, L$, relay, the received signal of (2) is first processed by the lth relay on a symbol-bysymbol basis, in order to generate a soft estimate, $\hat{x}_{k}[n]$ for $x_{k}[n]$ transmitted by the $k$ th source MT. Specifically, $r_{1}^{(k)}(t)$ is first input to a filter matched to the transmitted chip-waveform $\psi_{T_{\mathrm{c}}}(t)$, and then the matched-filter's output is sampled at the chip-rate, yielding

$$
\begin{array}{r}
y_{I \lambda}=\frac{1}{\sqrt{2 P_{l}^{(k)} N T_{\mathrm{c}}}} \int_{\lambda T_{\mathrm{c}}}^{(\lambda+1) T_{\mathrm{c}}} r_{l}^{(k)}(t) \psi_{T_{\mathrm{c}}}^{*}(t) \mathrm{d} t, \\
\lambda=0,1, \ldots, N-1
\end{array}
$$

where $\lambda T_{\mathrm{c}}$ is the sampling time-instant. Upon substituting (2) into (3), we obtain

$y_{l \lambda}=\frac{1}{\sqrt{N}} h_{l}^{(k)} x_{k}[n] c_{k \lambda}+N_{l \lambda}, \quad \lambda=0,1, \ldots, N-1$

where $N_{I \lambda}$ is the Gaussian noise component, which is given by

$$
N_{l \lambda}=\frac{1}{\sqrt{2 P_{l}^{(k)} N T_{\mathrm{c}}}} \int_{\lambda T_{\mathrm{c}}}^{(\lambda+1) T_{\mathrm{c}}} n_{l}^{(k)}(t) \psi_{T_{\mathrm{c}}}^{*}(t) \mathrm{d} t
$$

which has mean zero and a variance of $N_{0} / 2 E_{l}^{(k)}$ per dimension, where $E_{l}^{(k)}=P_{1}^{(k)} T$ represents the energy per symbol received by the lth relay from the $k$ th transmitter. Let us define

$$
\begin{aligned}
& \boldsymbol{y}_{l}=\left[y_{l 0}, y_{l 1}, \ldots, y_{l(N-1)}\right]^{\mathrm{T}} \\
& \boldsymbol{N}_{l}=\left[N_{l 0}, N_{l 1}, \ldots, N_{l(N-1)}\right]^{\mathrm{T}} \\
& \boldsymbol{c}_{k}=\frac{1}{\sqrt{N}}\left[c_{k 0}, c_{k 1}, \ldots, c_{k(N-1)}\right]^{\mathrm{T}}
\end{aligned}
$$


Then, it can be shown that we have

$$
y_{l}=c_{k} h_{l}^{(k)} x_{k}[n]+N_{l}
$$

When given the knowledge of $h_{l}^{(k)}$, it can be shown that the estimate $\hat{x}_{k}[n]$ to $x_{k}[n]$ can be readily obtained from (7), which can be expressed as

$$
\begin{aligned}
\hat{x}_{k}[n] & =\frac{1}{\left|h_{l}^{(k)}\right|^{2}}\left(h_{l}^{(k)}\right)^{*} \boldsymbol{c}_{k}^{\mathrm{T}} \boldsymbol{y}_{l}=\frac{1}{h_{l}^{(k)}} \boldsymbol{c}_{k}^{\mathrm{T}} \boldsymbol{y}_{l} \\
& =x_{k}[n]+\frac{1}{h_{l}^{(k)}} \boldsymbol{c}_{k}^{\mathrm{T}} \boldsymbol{N}_{l}
\end{aligned}
$$

Explicitly, $\hat{x}_{k}[n]$ contains noise in addition to the desired bit $x_{k}[n]$ to be relayed. Furthermore, it can be shown that we have

$$
\boldsymbol{s}_{k l}=\boldsymbol{E}\left[\left|\hat{x}_{k}[n]\right|^{2}\right]=1+\frac{1}{\left|h_{l}^{(k)}\right|^{2}} \times \frac{N_{0}}{E_{l}^{(k)}}
$$

After the estimation, $\hat{x}_{k}[n]$ is then spread and forwarded by the 1 th relay to the $\mathrm{BS}$ within the second time-slot of the $n$th symbol. Correspondingly, the transmitted complex baseband equivalent signal of the lth relay can be expressed as

$$
s_{l}^{(k)}(t)=\sqrt{\frac{2 P_{l t}^{(k)}}{s_{k l}}} \hat{x}_{k}[n] c_{l}^{(k)}(t), \quad l=1,2, \ldots, L
$$

where $P_{l \mathrm{t}}^{(k)}$ and $c_{l}^{(k)}(t)$ represent, respectively, the transmitted power per dimension and spread-spectrum signature waveform associated with the lth relay.

\subsection{Received signal at BS}

At the BS, the receiver can obtain observations for detecting $x_{k}[n]$ within both the first and second timeslots. Specifically, within the first time-slot of the $n$th symbol duration, the received complex baseband equivalent signal can be expressed as

$$
r_{0}(t)=\sqrt{2 P_{k r}} h_{0}^{(k)} x_{k}[n] c_{k}(t)+n(t)
$$

where $P_{k r}$ represents the power (per dimension) received from MT $k$, which is usually not the same as the transmitted power $P_{k t}$ because of the pathloss of the D-channel, $h_{0}^{(k)}$ represents the channel gain of the D-channel, while $n(t)$ denotes the Gaussian noise received at the $\mathrm{BS}$, which, again, has zero mean and a variance of $N_{0}$ per dimension.

By contrast, the received complex baseband equivalent signal by the BS during the second time-slot of the $n$th symbol duration can be expressed as

$$
\begin{aligned}
r_{1}(t)= & n(t)+\sum_{l=1}^{L} \sqrt{\frac{2 P_{l \mathrm{r}}^{(k)}}{\boldsymbol{s}_{k l}}} h_{r l}^{(k)} x_{k}[n] c_{l}^{(k)}(t) \\
& +\sum_{l=1}^{L} \sqrt{\frac{2 P_{l \mathrm{r}}^{(k)}}{\boldsymbol{S}_{k l}}} h_{r l}^{(k)}\left[\frac{\boldsymbol{c}_{k}^{\mathrm{T}} N_{l}}{h_{l}^{(k)}}\right] c_{l}^{(k)}(t)
\end{aligned}
$$

where $P_{l r}^{(k)}$ represents the power received by the BS from the 1 th relay of MT $k$. Due to the propagation pathloss, $P_{\mathrm{lr}}^{(k)}$ is also smaller than $P_{\mathrm{lt}}^{(k)}$ seen in $(10)$.

\subsection{Channel model}

The channel model considered includes both propagation pathloss and fast fading. We assume that the propagation pathloss satisfies the $\beta$ th power law and the pathloss, $L_{\mathrm{p}}(d)$, as a function of transmitterreceiver $(\mathrm{T}-\mathrm{R})$ separation distance $d$, can be expressed as [15]

$$
L_{\mathrm{p}}(\mathrm{d})(\mathrm{dB})=L_{\mathrm{s}}\left(\mathrm{d}_{0}\right)(\mathrm{dB})+10 \beta \log \left(\frac{d}{d_{0}}\right)
$$

where, $L_{\mathrm{s}}\left(d_{0}\right)$ is the pathloss measured at the reference distance $d_{0}, \beta$ is the pathloss exponent, which takes a typical value of 2 in free-space and 4 in cellular mobile systems.

In the context of the fast fading experienced by the signals transmitted from the source MT to the BS and relays, as well as the signals from the relays to the BS, we assume a generalised Nakagami-m fading channel model. Specifically in (12), let $h_{l}^{(k)}=\left|h_{l 1}^{(k)}\right| \mathrm{e}^{j \theta_{l 1}^{(k)}}$ and $h_{r l}^{(k)}=\left|h_{12}^{(k)}\right| \mathrm{e}^{j_{l 1}^{(k)}}$ for $1=1,2, \ldots, L$, where $\left|h_{l 1}^{(k)}\right|$, $\left|h_{12}^{(k)}\right|$ and $\theta_{11}^{(k)}, \theta_{12}^{(k)}$ denote the amplitudes and phases of the lth TR-channel and RB-channel, respectively. Then, the fading amplitude $\left|h_{l i}^{(k)}\right|, i=1,2$, obeys the Nakagami-m distribution with the probability density functions (PDF) given by [16, 17]

$$
f_{\left|h_{l i}^{(k)}\right|}(y)=\frac{2 m_{l i}^{m_{l i} y^{2 m_{l i}-1}}}{\Gamma\left(m_{l i}\right) \Omega_{l i}} \mathrm{e}^{-m_{l i} y^{2} / \Omega_{l i}}, \quad i=1,2
$$

where $\Gamma(\cdot)$ is the gamma function [18], $\Omega_{l i}=E\left[\left|h_{l i}^{(k)}\right|^{2}\right]$ and $m_{l i}$ represents the fading parameter of the lth TRchannel when $i=1$ or the lth RB-channel if $i=2$. Note that $h_{0}^{(k)}=\left|h_{0}^{(k)}\right| \mathrm{e}^{j \theta_{0}^{(k)}}$, where $\left|h_{0}^{(k)}\right|$ and $\theta_{0}^{(k)}$ denote the amplitude and phase of the D-channel. The PDF of $\left|h_{0}^{(k)}\right|$ can be readily obtained from (14) with $m_{l i}$ replaced by $m_{0}$ and $\Omega_{l i}$ by $\Omega_{0}$. Furthermore, the phases $\theta_{0}$ and $\theta_{l i}$ for $l=1,2, \ldots, L$ are assumed to 
be the independent identically distributed (iid) random variables uniformly distributed in $[0,2 \pi)$.

Note that, we introduce the channel model having the Nakagami-m distribution as shown in (14) because, in the considered relay-assisted DS-CDMA system, the Dchannel, TR-channel and the RB-channel may experience different fading. For example, when the relays are chosen in the line-of-sight (LoS) area of the BS, the distance from the source transmitter to the relays/BS may be significantly higher than the distance from the relays to the BS. In this case, the D-channel and TR-channels may experience Rayleigh fading corresponding to $m_{l i}=1$ in (14), whereas the RB-channel experiences Rician fading corresponding to $m_{l i}>1$ in (14).

\section{Signal detection}

In this section, we consider the signal combining and detection, in order to recover the transmitted information by the source MT. There are many multiuser detection and array processing schemes [19, 20], which may be modified for our DS-CDMA system using relay diversity. As two examples, in this contribution we consider an SUC scheme based on the MRC and a MUC scheme, which is derived under the MSINR criterion. Let us first represent the signals received by the BS within the first and second timeslots.

\subsection{Received signal representation at BS}

As at the relays, the received signal at the BS is first filtered by a chip-waveform matched-filter and is then sampled at chip-rate in order to provide the detector observation samples. Since $x_{k}[n]$ is transmitted by two time-slots, each of which is associated with an $N$-length spreading sequence, hence the BS receiver can obtain a total of $2 \mathrm{~N}$ samples for detecting $x_{k}[n]$. Let $y=\left[y_{0}^{\mathrm{T}}, y_{1}^{\mathrm{T}}\right]^{\mathrm{T}}$ contain the $2 \mathrm{~N}$ observation samples, where $y_{i}=\left[y_{i 0}, y_{i 1}, \ldots, y_{i(N-1)}\right]^{\mathrm{T}}, i=0,1$. It can be shown that, after the normalization using $1 / \sqrt{ }\left(2 P_{k \mathrm{r}} N\right) T_{\mathrm{c}}, y_{i \lambda}$ can be expressed as

$$
\begin{gathered}
y_{i \lambda}=\frac{1}{\sqrt{2 P_{k \mathrm{r}} N T_{\mathrm{c}}}} \int_{\lambda T_{\mathrm{c}}}^{(\lambda+1) T_{\mathrm{c}}} r_{i}(t) \psi_{T_{\mathrm{c}}}^{*}(t) \mathrm{d} t, \quad i=0,1 ; \\
\lambda=0,1, \ldots, N-1
\end{gathered}
$$

Let us define $\zeta_{k l}=P_{l \mathrm{r}}^{(k)} /\left(\boldsymbol{s}_{k l} P_{k \mathrm{r}}\right)$, where $\boldsymbol{s}_{k l}$ is given in (9). Then, upon substituting (11) and (12) into the above equation, we obtain

$$
\begin{aligned}
y_{0 \lambda}= & \frac{1}{\sqrt{N}} h_{0}^{(k)} c_{k \lambda} x_{k}[n]+n_{0 \lambda} \\
y_{1 \lambda}= & \frac{1}{\sqrt{N}} \sum_{l=1}^{L} \sqrt{\zeta_{k l}} h_{r l}^{(k)} c_{l \lambda}^{(k)} x_{k}[n] \\
& +\frac{1}{\sqrt{N}} \sum_{l=1}^{L} \sqrt{\zeta_{k l}} h_{r l}^{(k)} c_{l \lambda}^{(k)}\left[\frac{\left.c_{k}^{\mathrm{T}} \boldsymbol{N}_{l}\right]}{h_{l}^{(k)}}\right]+n_{1 \lambda}
\end{aligned}
$$

for $\lambda=0,1, \ldots, N-1$, where $n_{i \lambda}, i=0,1$ is an independent Gaussian random variable with zero mean and a variance of $N_{0} / 2 E_{k r}$ per dimension, where $E_{k \mathrm{r}}=P_{k \mathrm{r}} T_{b}$ represents the average energy per symbol received from the $\mathrm{D}$-channel.

Let

$$
\begin{aligned}
& \boldsymbol{c}_{k l}=\frac{1}{\sqrt{N}}\left[c_{l 0}^{(k)}, c_{l 1}^{(k)}, \ldots, c_{l(N-1)}^{(k)}\right]^{\mathrm{T}} \\
& \boldsymbol{n}_{i}=\left[n_{i 0}, n_{i 1}, \ldots, n_{i(N-1)}\right]^{\mathrm{T}}, \quad i=0,1
\end{aligned}
$$

Then, it can be shown that, after ignoring the superscript $k$ for convenience, $y$ can be expressed as

$$
\begin{aligned}
& y=\left[\begin{array}{c}
c_{k} h_{0} \\
\sum_{l=1}^{L} c_{k l} \sqrt{\zeta_{k l}} h_{r l}
\end{array}\right] x_{k}[n] \\
& +\left[\sum_{l=1}^{L} c_{k l} \sqrt{\zeta_{k l}} h_{r l}\left[\frac{c_{k}^{\mathrm{T}} \boldsymbol{N}_{l}}{h_{l}}\right]\right]+\left[\begin{array}{l}
\boldsymbol{n}_{0} \\
\boldsymbol{n}_{1}
\end{array}\right]
\end{aligned}
$$

Furthermore, the above equation can be rewritten as

$$
y=\boldsymbol{C}_{k} \boldsymbol{A}_{k} \boldsymbol{h}_{k} X_{k}[n]+\underbrace{\boldsymbol{C}_{k r} \overline{\boldsymbol{A}}_{k} \boldsymbol{H}_{k r}\left(\boldsymbol{I}_{L} \otimes \boldsymbol{c}_{k}^{\mathrm{T}}\right) \boldsymbol{n}_{t r}+\boldsymbol{n}_{r}}_{\boldsymbol{n}_{I}}
$$

where $\otimes$ represents the Kronecker product [20] operation and the other arguments in (20) are given as follows

$$
\begin{gathered}
\boldsymbol{C}_{k}=\left[\begin{array}{ccccc}
\boldsymbol{c}_{k} & \mathbf{0} & \mathbf{0} & \ldots & \mathbf{0} \\
\mathbf{0} & \boldsymbol{c}_{k 1} & \boldsymbol{c}_{k 2} & \ldots & \boldsymbol{c}_{k L}
\end{array}\right] \\
\boldsymbol{A}_{k}=\operatorname{diag}\left\{1, \sqrt{\zeta_{k 1}}, \sqrt{\zeta_{k 2}}, \ldots, \sqrt{\zeta_{k L}}\right\} \\
\boldsymbol{h}_{k}=\left[h_{0}, h_{r 1}, h_{r 2}, \ldots, h_{r L}\right]^{\mathrm{T}}
\end{gathered}
$$




$$
\begin{aligned}
& \boldsymbol{C}_{k r}= {\left[\begin{array}{cccc}
\mathbf{0} & \mathbf{0} & \ldots & \mathbf{0} \\
\boldsymbol{c}_{k 1} & \boldsymbol{c}_{k 2} & \ldots & \boldsymbol{c}_{k L}
\end{array}\right] } \\
& \boldsymbol{H}_{k r}=\operatorname{diag}\left\{\frac{h_{r 1}}{h_{1}}, \frac{h_{r 2}}{h_{2}}, \ldots, \frac{h_{r L}}{h_{L}}\right\} \\
& \mathbf{n}_{t r}=\left[\boldsymbol{N}_{1}^{\mathrm{T}}, \boldsymbol{N}_{2}^{\mathrm{T}}, \ldots, \boldsymbol{N}_{L}^{\mathrm{T}}\right]^{\mathrm{T}} \\
& \mathbf{n}_{r}=\left[\mathbf{n}_{0}^{\mathrm{T}}, \mathbf{n}_{1}^{\mathrm{T}}\right]^{\mathrm{T}}
\end{aligned}
$$

Furthermore, in (20), $\bar{A}_{k}$ is the $(L \times L)$-dimensional diagonal matrix, which is obtained from $A_{k}$ by removing the first element 1 .

With the assistance of (20), let us now consider the detection of $x_{k}[n]$ transmitted by the source MT using either the MRC-assisted SUC or the MSINR-assisted MUC.

\subsection{Maximal ratio combining}

In the context of the MRC-assisted SUC, the received signal vector $y$ of (20) is first despread using $\boldsymbol{C}_{k}^{\mathrm{T}}$, yielding

$$
\bar{y}=C_{k}^{\mathrm{T}} y
$$

where $\bar{y}=\left[\bar{y}_{0}, \bar{y}_{1}, \ldots, \bar{y}_{L}\right]^{\mathrm{T}}$. After the despreading, it can be shown that the lth component of $\bar{y}$ can be expressed as

$$
\bar{y}_{l}=\left\{\begin{array}{l}
h_{0} x_{k}[n]+c_{k}^{\mathrm{T}} n_{0}, \quad \text { if } l=0 \\
\sqrt{\zeta_{k l}} h_{r l} x_{k}[n]+\sqrt{\frac{\zeta_{k l} h_{r l}}{h_{l}} c_{k}^{\mathrm{T}} \boldsymbol{N}_{l}+\boldsymbol{c}_{k l}^{\mathrm{T}} \boldsymbol{n}_{1}+I_{\mathrm{RI}}} \\
\text { if } l=1,2, \ldots, L
\end{array}\right.
$$

where $I_{\mathrm{RI}}$ represents the inter-relay interference.

Since the MRC-assisted SUC scheme does not make use of the knowledge from the interfering relays, it can only treat the inter-relay interference as noise. Let us assume that the second-order moment of the $I_{\mathrm{RI}}$ in (29) is given by $\sigma_{\text {IRI }}^{2}$, it can be readily shown that the weights for combining the direct and the relayed signals are given by

$$
w_{l}=\left\{\begin{array}{lc}
\left(\frac{N_{0}}{E_{k r}}\right)^{-1} h_{0}^{*}, & \text { for } l=0 \\
\left(\frac{\zeta_{k l}\left|h_{r l}\right|^{2}}{\left|h_{l}\right|^{2}} \frac{N_{0}}{E_{l}^{(k)}}+\frac{N_{0}}{E_{k r}}+\sigma_{\mathrm{IRI}}^{2}\right)^{-1} \sqrt{\zeta_{k l}} h_{r l}^{*}, & \text { for } l=1,2, \ldots, L
\end{array}\right.
$$

respectively. Correspondingly, the decision variable $z_{k}[n]$ for $x_{k}[n]$ is given by

$$
z_{k}[n]=\sum_{l=0}^{L} w_{\nu} \bar{y}_{l}
$$

Note that, the above-derived combining scheme shown in (31) maximises the output SNR, when there exists no inter-relay interference or when the interrelay interference is indeed Gaussian. However, the inter-relay interference is usually not Gaussian. In this case, the MRC-assisted SUC using the weights shown in (30) is not optimum and the inter-relay interference significantly degrades the achievable performance.

Upon substituting (29) and (30) into (31), it can be shown that the SNR can be expressed as

$$
\begin{aligned}
\gamma= & \left(\frac{N_{0}}{E_{k r}}\right)^{-1}\left|h_{0}\right|^{2}+\sum_{l=1}^{L}\left(\frac{\zeta_{k l}\left|h_{r l}\right|^{2}}{\left|h_{l}\right|^{2}} \frac{N_{0}}{E_{l}^{(k)}}+\frac{N_{0}}{E_{k r}}+\sigma_{\mathrm{IRI}}^{2}\right)^{-1} \\
& \times \zeta_{k l}\left|h_{r l}\right|^{2} \\
= & \frac{\left|h_{0}\right|^{2} E_{k r}}{N_{0}}+\sum_{l=1}^{L}\left(\frac{N_{0}}{\left|h_{l}\right|^{2} E_{l}^{(k)}}+\frac{N_{0} / E_{k r}+\sigma_{\mathrm{IRI}}^{2}}{\zeta_{k l}\left|h_{r l}\right|^{2}}\right)^{-1} \\
= & \gamma_{0}+\sum_{l=1}^{L}\left(\frac{1}{\gamma_{l}}+\frac{1}{\gamma_{r l}}\right)^{-1}
\end{aligned}
$$

where

$$
\begin{aligned}
\gamma_{0} & =\frac{\left|h_{0}\right|^{2} E_{k r}}{N_{0}} \\
\gamma_{l} & =\frac{\left|h_{1}\right|^{2} E_{l}^{(k)}}{N_{0}}, \quad l=1,2, \ldots, L \\
\gamma_{r l} & =\frac{\zeta_{k l}\left|h_{r l}\right|^{2}}{N_{0} / E_{k r}+\sigma_{\mathrm{IRI}}^{2}}, \quad l=1,2, \ldots, L
\end{aligned}
$$

and $\gamma_{0}, \gamma_{1}$ and $\gamma_{r l}$ represent the instantaneous SNRs of the D-channel, the lth TR-channel and the lth RBchannel, respectively.

Furthermore, when a DS-CDMA system using binary phase-shift keying (BPSK) baseband modulation is assumed, then, upon applying the Gaussian approximation of the inter-relay interfering signals, the BER conditioned on the instantaneous SNRs can be 
expressed as

$$
\begin{aligned}
P_{b}\left(\gamma_{0},\left\{\gamma_{l}\right\},\left\{\gamma_{r l}\right\}\right) & =Q(\sqrt{2 \gamma}) \\
Y & =Q\left(\sqrt{2 \gamma_{0}+2 \sum_{l=1}^{L}\left(\frac{1}{\gamma_{l}}+\frac{1}{\gamma_{r l}}\right)^{-1}}\right)
\end{aligned}
$$

Based on (34), the average BER for some special cases may be approximately estimated using the average BER equations in [9-11], which have been derived based on (34) associated with assuming that there exists no interference among the relays. Therefore the average BER equations in [9-11] may be employed for evaluating the BER of the proposed scheme, either when orthogonal spreading sequences are employed or when the number of relays is high yielding that the Gaussian approximation is accurate. However, for the general case considered in this contribution, where different channels may experience different fading and the relays interfere with each other, it is extremely difficult to derive the closed-form average BER expression. Furthermore, since usually only a small number of relays are employed for assisting the source MT, the inter-relay interference cannot be accurately approximated by a Gaussian variable. Therefore in this contribution Monte-Carlo simulation approaches are employed for obtaining the BER results in Section 4.

Let us now consider the MSINR-assisted MUC, which is capable of efficiently suppressing the interrelay interference.

\subsection{MSINR combining}

For the sake of simplicity, we re-write the observation vector of (20) as

$$
\boldsymbol{y}=\overline{\boldsymbol{h}}_{k} x_{k}[n]+\boldsymbol{n}_{I}
$$

with

$$
\begin{aligned}
\overline{\boldsymbol{h}}_{k} & =\boldsymbol{C}_{k} \boldsymbol{A}_{k} \boldsymbol{h}_{k} \\
\boldsymbol{n}_{I} & =\boldsymbol{C}_{k r} \overline{\boldsymbol{A}}_{k} \boldsymbol{H}_{k r}\left(\boldsymbol{I}_{L} \otimes \boldsymbol{c}_{k}^{\mathrm{T}}\right) \boldsymbol{n}_{t r}+\boldsymbol{n}_{r}
\end{aligned}
$$

The maximum SINR-assisted MUC scheme maximises the output SINR with the aid of the knowledge about MT $k$ as well as its relays, that is, the BS receiver employs the knowledge about the spreading codes and channels of both the $k$ th MT and its relays.
Let $\boldsymbol{w}_{o}$ be the $2 \mathrm{~N}$-length weight vector obtained in MSINR sense. Then the decision variable for $z_{k}[n]$ can be expressed as

$$
z_{k}[n]=\boldsymbol{w}_{o}^{H} \boldsymbol{y}=\boldsymbol{w}_{o}^{H} \overline{\boldsymbol{h}}_{k}{ }_{k}[n]+\boldsymbol{w}_{o}^{H} \boldsymbol{n}_{I}
$$

The optimum weight vector $\boldsymbol{w}_{o}$ in MSINR sense is chosen as

$$
\boldsymbol{w}_{o}=\arg \max _{\boldsymbol{w}}\left\{\operatorname{SINR}=\frac{\left\|\boldsymbol{w}^{H} \overline{\boldsymbol{h}}_{k}\right\|^{2}}{\boldsymbol{w}^{H} \boldsymbol{R}_{I} \boldsymbol{W}}\right\}
$$

where $R_{I}=E\left[\boldsymbol{n}_{I} \boldsymbol{n}_{I}^{H}\right]$ is the covariance matrix of $\boldsymbol{n}_{I}$. It is well-known that the optimum weight vector of $\boldsymbol{w}_{o}$ for the MSINR-assisted MUC is given by [21]

$$
\boldsymbol{w}_{o}=\mu \boldsymbol{R}_{I}^{-1} \overline{\boldsymbol{h}}_{k}=\mu \boldsymbol{R}_{I}^{-1} \boldsymbol{C}_{k} \boldsymbol{A}_{k} \boldsymbol{h}_{k}
$$

Furthermore, it can be shown that, when the MSINR solution is achieved, the corresponding $\mathrm{SNR}$ is given by [21]

$$
\operatorname{MSINR}=\overline{\boldsymbol{h}}_{k}^{H} \boldsymbol{R}_{I}^{-1} \overline{\boldsymbol{h}}_{k}
$$

and when the BPSK modulation is employed, the BER conditioned on the fading can be expressed as [18]

$$
P_{b}\left(h_{0},\left\{h_{r l}\right\},\left\{h_{l}\right\}\right)=Q\left(\sqrt{2 \overline{\boldsymbol{h}}_{k}^{H} \boldsymbol{R}_{I}^{-1} \overline{\boldsymbol{h}}_{k}}\right)
$$

Again, it is highly difficult to derive the closed-form BER expression based on (40) and, hence, simulation approaches are invoked to obtain the results in Section 4.

\section{Performance results}

In this section, we provide a range of simulation results in order to illustrate the single-user BER performance of the DS-CDMA system using relay diversity, when the MRC-assisted SUC of (31) or MSINR-assisted MUC of (37) is employed for combining the received signals. Specifically, in our simulations, we assumed that the BPSK baseband modulation scheme was employed. In most cases, we assumed that the relays were chosen from the vicinity area of the BS. Correspondingly, as we mentioned previously in Section 2.4, in our simulations we assumed that both the D-channel and TR-channels were modelled as independent Rayleigh fading channels, whereas the RB-channels were modelled as Nakagami-m fading channels associated with a fading parameter higher than one. The main objectives of the results shown in this section include 
- to illustrate the achievable BER performance of the single-user DS-CDMA using various number of relays,

- to show the impact of the relays' locations on the achievable BER performance and

- to show the impact of the power allocation between the source MT and the relays on the achievable BER performance.

Note that, in order to carry out a fair comparison for the power-efficiency, we assume that the total transmission power consumed per symbol is the same for both our proposed relay system and the direct system, which does not use relays, that is, we assume that $P_{0}=P_{k t}+L P_{l t}^{(k)}$, where $P_{0}$ is the total power radiated by a transmitter in the direct system. In our simulations a parameter $\alpha$ was introduced, which counts for the power allocation. Specifically, in our proposed relay-assisted system, $\alpha P_{0}$ is allocated to the first time-slot for the source MT to transmit signals to the BS and relays. By contrast, the amount of $(1-\alpha) P_{0}$ is allocated to the second time-slot for the relays to forward the information to the BS. Furthermore, for convenience, in our simulations all the distances in the relay-assisted system are normalised by the distance $d_{T B}$ between the source transmitter and BS. We assume that the distance between the source transmitter and BS is unity, whereas the distance between the cluster of relays and BS is $\delta$, where $0<\delta<1$.

In Fig. 2, we evaluated the BER against $(\alpha, \delta)$ performance, when the D-channel and TR-channels experienced Rayleigh fading, whereas the RB-channels experienced Nakagami-m fading associated with $m_{12}=2$ for $L=3$. In our simulations, we assumed that the pathloss exponent corresponding to the largescale-fading was $\beta=3$ and the SNR per bit was $E_{b} / N_{0}=4 \mathrm{~dB}$. It can be seen from Fig. 2 that increasing $\alpha$, that is, assigning more power to the

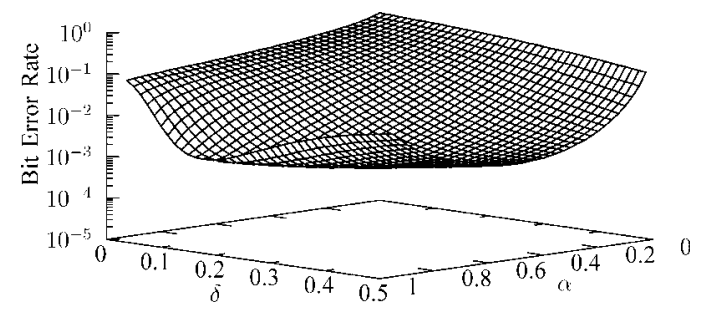

Figure 2 BER against the parameters $(\alpha, \delta)$ performance for the relay-assisted DS-CDMA using $m$-sequences and MSINR-assisted MUC, when the D-channel and the TRchannels experience Rayleigh fading, whereas the RBchannels experience Nakagami-m fading associated with $m_{12}=2$

In this figure, we assumed that $L=3, E_{b} / N_{0}=4 \mathrm{~dB}$ and that the pathloss exponent was $\beta=3$

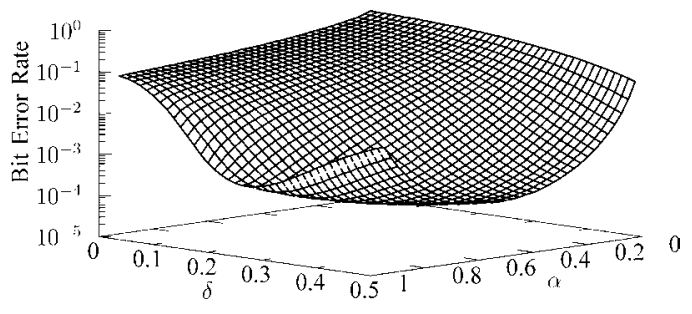

Figure 3 BER against the parameters $(\alpha, \delta)$ performance for the relay-assisted DS-CDMA using $m$-sequences and MSINR-assisted MUC, when the D-channel and the TRchannels experience Rayleigh fading, whereas the RBchannels experience Nakagami-m fading associated with $m_{12}=2$

In this figure, we assumed that $\mathrm{L}=3, \mathrm{E}_{\mathrm{b}} / \mathrm{N}_{0}=4 \mathrm{~dB}$ and that the pathloss exponent was $\beta=4$

transmitter in the first time slot, generally results in an improved BER performance. Furthermore, when increasing the value of $\delta$, which implies that the relays move from the BS toward the transmitter, the BER performance improves. However, when the values of $\alpha$ and $\delta$ are too high, for example, when $\alpha$ and $\delta$ are in the range of $[0.8,0.95]$ and $[0.2,0.5]$, respectively, the BER performance degrades. Therefore as seen in Fig. 2, for any a given value of $\alpha$, there may exist an optimum value of $\delta$, which results in the lowest BER. Vice versa, for any given value of $\delta$, there exists an optimum value of $\alpha$, implying the optimum power allocation, which also results in the lowest BER.

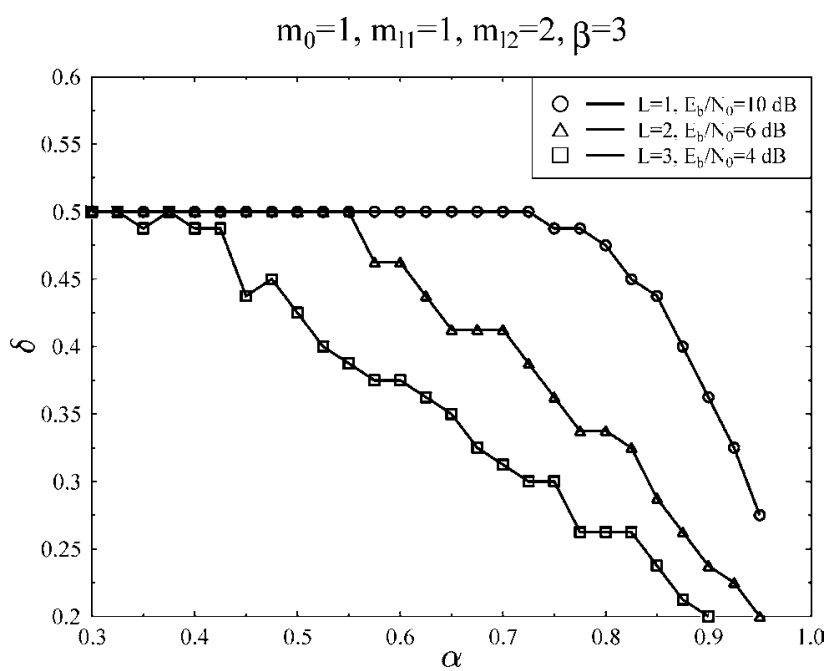

Figure 4 The values of $\alpha$ and $\delta$, which result in the minimum BER for the relay-assisted DS-CDMA system using $m$-sequences and MSINR-assisted MUC, when the $D$-channel and the TR-channels experience Rayleigh fading, whereas the RB-channels experience Nakagami-m fading associated with $m_{12}=2$

Other parameters are $E_{b} / N_{0}=10 d B$ for $L=1, E_{b} / N_{0}=6 d B$ for $\mathrm{L}=2$ and $\mathrm{E}_{\mathrm{b}} / \mathrm{N}_{0}=4 \mathrm{~dB}$ for $\mathrm{L}=3$ and the pathloss exponent is $\beta=3$ 


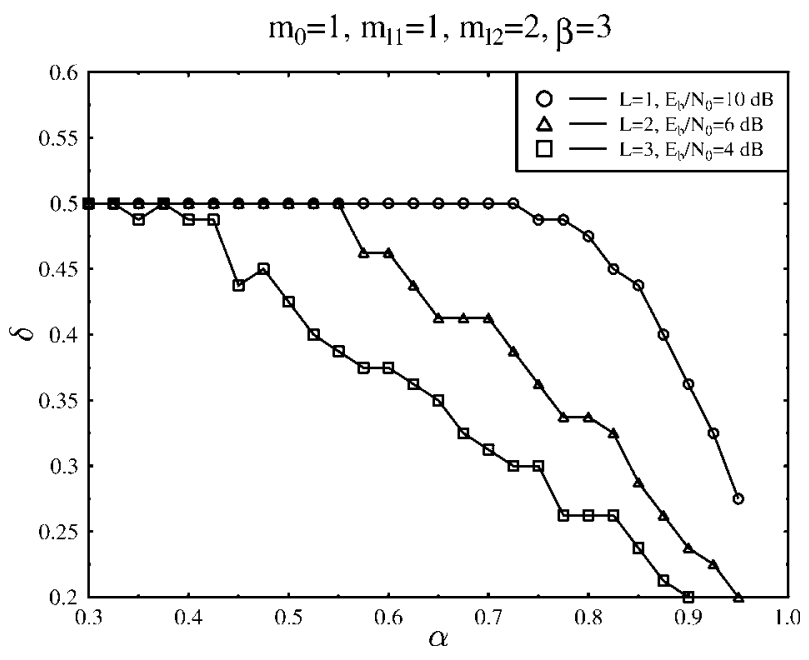

Figure 5 Values of $\alpha$ and $\delta$, which result in the minimum $B E R$ for the relay-assisted DS-CDMA system using $m$ sequences and MSINR-assisted MUC, when the D-channel and the TR-channels experience Rayleigh fading, whereas the RB-channels experience Nakagami-m fading associated with $m_{12}=2$

Other parameters are $E_{b} / N_{0}=10 \mathrm{~dB}$ for $L=1, E_{b} / N_{0}=6 \mathrm{~dB}$ for $L=2$ and $E_{b} / N_{0}=4 \mathrm{~dB}$ for $L=3$ and the pathloss exponent is $\beta=4$

In Fig. 3, we evaluated the BER against $(\alpha, \delta)$ performance of the relay-assisted DS-CDMA system, when the D-channel and the TR-channels experienced Rayleigh fading, whereas the RB-channels experienced Nakagami- $m$ fading associated with $m_{12}=2$ for $L=3$. The parameters used in our simulations in Fig. 3 were the same as those employed for Fig. 2, except for the pathloss exponent, which was $\beta=4$ for Fig. 3. From the results of Figs. 2 and 3, it can be observed that for the same number of relays, the same $E_{b} / N_{0}$ value and the same value of $\alpha$, the optimum value of $\delta$ increases when the pathloss exponent increases from 3 to 4. This observation indicates that, when the pathloss exponent increases, the relays should be chosen from the area, which has a similar distance from both the transmitter and the BS, in order to minimise the BER.

Figs. 4 and 5 show the corresponding $(\alpha, \delta)$ values that achieved the minimum BER for the relay-assisted DS-CDMA systems using $m$-sequences and MSINRassisted MUC. In our simulations for both figures we assumed that the D-channel and the TR-channels experienced the Rayleigh fading, whereas the RBchannels experienced the Nakagami-m fading associated with $m_{12}=2$. The other parameters employed in our simulations were $E_{b} / N_{0}=10 \mathrm{~dB}$ for $L=1, E_{b} / N_{0}=6 \mathrm{~dB}$ for $L=2$ and $E_{b} / N_{0}=4 \mathrm{~dB}$ for $L=3$. The different parameter used for Figs. 4 and 5 was the pathloss exponent, which was $\beta=3$ for
Fig. 4 and $\beta=4$ for Fig. 5. From the results of these figures, we may derive the following observations. First, the optimum location of the cluster of relays is not necessarily in the middle between the source MT and BS. Secondly, for given $L$ and SNR values, when the relays are chosen from a different area, the power allocated to the first and second time-slots should be adjusted, in order to achieve a better BER performance. Thirdly, when the relays are chosen from an area that is closer to the BS, more power can be allocated to the first time-slot for achieving the minimum possible BER. Furthermore, when comparing Fig. 4 with Fig. 5, we can see that the $(\alpha$, $\delta$ ) curves move toward the righthand side, when the pathloss exponent $\beta$ increases from $\beta=3$ of Fig. 4 to $\beta=4$ of Fig. 5. This phenomenon implies that, when the pathloss exponent $\beta$ increases, more power should be allocated to the first time-slot for broadcasting the source information to the relays and BS, in order to achieve the minimum BER.

Finally, in Figs. 6 and 7, we evaluated the BER against the average SNR per bit performance for the relayassisted DS-CDMA system associated with using the MRC-assisted SUC (Fig. 6) and MSINR-assisted MUC (Fig. 7), when assuming that the D-channel and the TR-channels experienced the Rayleigh fading, while the RB-channels experienced the Nakagami-m fading associated with $m_{12}=2$. In our simulations, we assumed random spreading sequences, and the parameters for both figures were $\alpha=0.9, \delta=0.3$ and $\beta=4$. As shown by the results in Figs. 6 and 7 ,

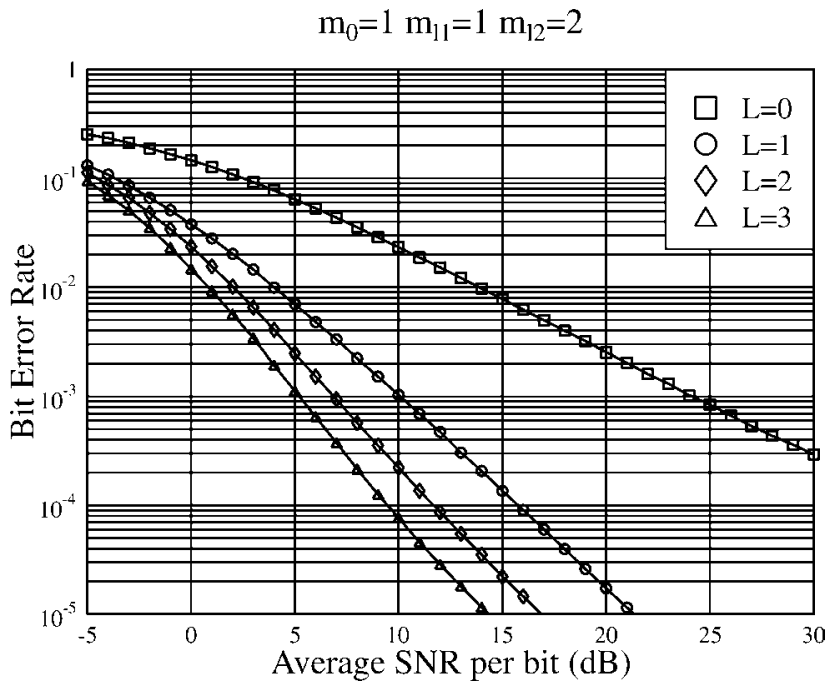

Figure 6 BER against SNR per bit performance of the relayassisted DS-CDMA using random sequences and MRCassisted SUC, when the D-channel and the TR-channels experience Rayleigh fading, whereas the RB-channels experience Nakagami-m fading associated with $m_{12}=2$ for $I=1,2,3$

Other parameters assumed were $\alpha=0.9, \delta=0.3$ and $\beta=4$ 


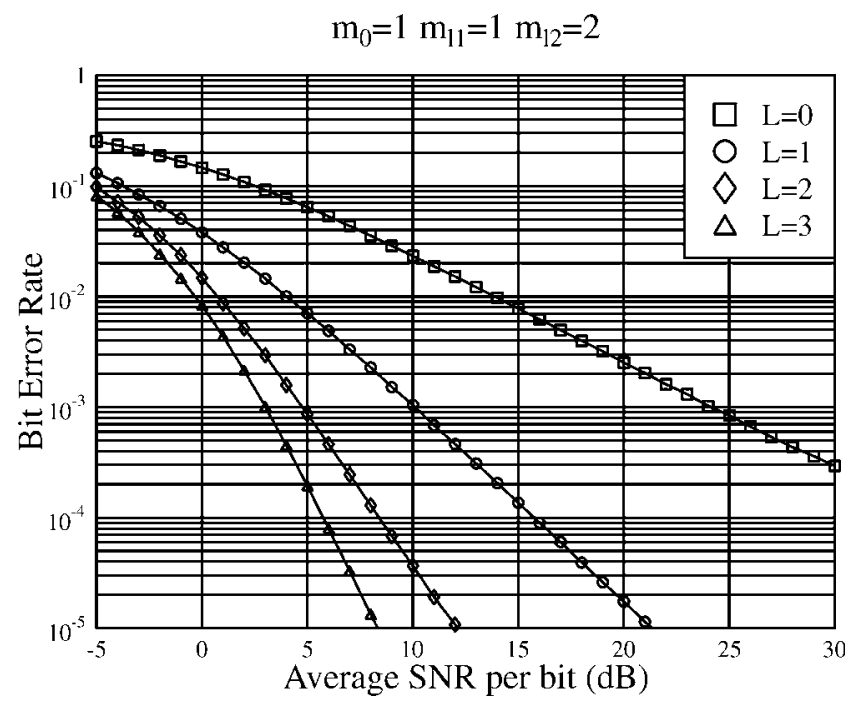

Figure 7 BER againts SNR per bit performance of the relayassisted DS-CDMA using random sequences and MSINRassisted MUC, when the D-channel and the TR-channels experience Rayleigh fading, whereas the RB-channels experience Nakagami- $m$ fading associated with $m_{12}=2$ for $I=1,2,3$

Other parameters assumed were $\alpha=0.9, \delta=0.3$ and $\beta=4$

the BER performance of the DS-CDMA is significantly improved, when the source MT can be assisted by more relays. However, when the BS employs the MRC-SUC scheme, the interference among the relays degrades significantly the achievable BER performance. This observation becomes explicit when comparing the results in Fig. 6 with that in Fig. 7, where the interference among the relays is efficiently suppressed with the aid of the MSINR-MUC. Specifically, as shown in Figs. 6 and 7, for the case of $L=3$, the MSINR-MUC is capable of achieving a BER of $10^{-5}$ at a SNR of $8 \mathrm{~dB}$, whereas the MRCSUC requires a SNR of $14.5 \mathrm{~dB}$ in order to achieve the BER of $10^{-5}$.

\section{Conclusions}

In this contribution, we have investigated the performance of a DS-CDMA using relay-diversity, when communicating over the generalised Nakagami-m fading channels associated with considering the propagation pathloss. We have focused our attention on the case of single source MT assisted by multiple relays, so that we can gain insight into the achievable BER performance, when the DS-CDMA system supports multiple source MTs associated with employing advanced multiuser detections. Our study and simulation results show that the lowest achievable BER of the DS-CDMA using relay diversity depends on the locations of the relays as well as on the power-allocation among the source MT and relays. When the relays of a source MT are chosen from a different area, the power-allocation should also be adjusted correspondingly in order to achieve the minimum BER. When optimum power-allocation is applied, the BER performance of the DS-CDMA can be significantly improved, when increasing the number of relays assisting the source MT. Furthermore, when there exists interference among the relays, a MUC scheme is required for suppressing the interference and achieving a better BER performance. Our future work will endeavour to extend our research in this contribution to the DS-CDMA systems using relay diversity, when multiple source MTs are considered.

\section{Acknowledgment}

The author would like to acknowledge with thanks the financial assistance from EPSRC of UK.

\section{References}

[1] NABAR R.U., BÖLCSKEI H.: 'Space-time signal design for fading relay channels'. Proc. IEEE Globecom, December 2003, vol. 4, pp. 1952-1956

[2] TAROKH V., JAFARKHANI H., CALDERBANK A.R.: 'Space-time block coding for wireless communications: performance results', IEEE J. Sel. Areas Commun., 1999, 17, pp. 451-460

[3] GoldSMith A., JAfAR S.A., JindAL N., ET AL.: 'Capacity limits of MIMO channels', IEEE J. Sel. Areas Commun., 2003, 21, pp. 684-702

[4] YANG L.-L.: 'MIMO assisted space-code-division multipleaccess: linear detectors and performance over multipath fading channels', IEEE J. Sel. Areas Commun., 2006, 24, (1), pp. 121-131

[5] SENdonaris A., ERKIP E., AAZHANG B.: 'Increasing uplink capacity via user cooperation diversity'. Proc. IEEE Int. Symp. Information Theory, Cambridge, MA, August 1998, p. 56

[6] Sendonaris A., ERKIP E., AAZHANG B.: 'User cooperation diversity-Part I and II', IEEE Trans. Commun., 2003, 51, pp. 1927-1938

[7] LANeman J.N., TSE D.N.C., WORnell G.W.: 'Cooperative diversity in wireless networks: efficient protocols and outage behavior', IEEE Trans. Inf. Theory, 2004, 50, pp. 3062-3080

[8] BLETSAS A., KHISTI A., REED D., ET AL.: 'A simple cooperative diversity method based on network path selection', IEEE J. Sel. Areas Commun., 2006, 24, (3), pp. 659-672

[9] ANGHEL P.A., KAVEH M.: 'Exact symbol error probability of a cooperative network in a Rayleigh fading environment', IEEE Trans. Wirel. Commun., 2004, 3, pp. 1416-1421 
[10] HASNA M.O., ALOUINI M.S.: 'Harmonic mean and end-toend performance of transmission systems with relays', IEEE Trans. Commun., 2004, 52, pp. 130-135

[11] HASNA M.O., ALOUINI M.S.: 'End-to-end performance of transmission systems with relays over Rayleigh-fading channels', IEEE Trans. Wirel. Commun., 2003, 2, pp. 1126-1131

[12] BOYER J., FALCONER D., YANIKOMEROGLU h.: 'Multihop diversity in wireless relaying channels', IEEE Trans. Commun., 2004, 52, (10), pp. $1820-1830$

[13] CAO Y., VOJCIC B.: 'MMSE multiuser detection for cooperative diversity CDMA systems', IEEE Commun. Soc., 2004, 1, pp. 42-47

[14] VENTURINO L., WANG X., LOPS M.: 'Multiuser detection for cooperative networks and performance analysis', IEEE Trans. Signal Process., 2006, 54, pp. 3315-3329

[15] RAPPAPORT T.S.: 'Wireless Communications: Principles and Practice' (Prentice Hall, Upper Saddle River, NJ, 1996)
[16] NAKAgAMI N.: 'The $m$-distribution, a general formula for intensity distribution of rapid fading' in HoffMan W.G. (Ed.) Statistical Methods in Radio Wave Propagation' (Pergamon, Oxford, England, 1960)

[17] YANG L.-L., HANZO L.: 'Performance of generalized multicarrier DS-CDMA over Nakagami- $m$ fading channels', IEEE Trans. Commun., 2002, 50, pp. 956-966

[18] PROAKIS J.G.: 'Digital communications' (McGraw-Hill, New York, 1995)

[19] Verdú S.: 'Multiuser Detection' (Cambridge University Press, Cambridge, UK, 1998)

[20] TREES H.L.V.: 'Optimum array processing' (Wiley Interscience, 2002)

[21] COMPton R.T.J.: 'Adaptive antennas: concepts and performance' (Prentice-Hall, Englewood Cliffs, New Jersey, USA, 1996) 